OPEN ACCESS

Edited by:

Takao K. Hensch,

Harvard University, USA

Reviewed by:

Fadi A. Issa

East Carolina University, USA Marta Costa,

University of Cambridge, UK

*Correspondence:

Mark Stopfer

stopferm@mail.nih.gov

Received: 19 January 2017 Accepted: 10 April 2017

Published: 03 May 2017

Citation:

Shimizu K and Stopfer M (2017) A Population of Projection Neurons that Inhibits the Lateral Horn but Excites the Antennal Lobe through Chemical Synapses in Drosophila. Front. Neural Circuits 11:30. doi: 10.3389/fncir.2017.00030

\section{A Population of Projection Neurons that Inhibits the Lateral Horn but Excites the Antennal Lobe through Chemical Synapses in Drosophila}

\author{
Kazumichi Shimizu and Mark Stopfer * \\ National Institute of Child Health and Human Development, National Institutes of Health, Bethesda, MD, USA
}

In the insect olfactory system, odor information is transferred from the antennal lobe (AL) to higher brain areas by projection neurons (PNs) in multiple AL tracts (ALTs). In several species, one of the ALTs, the mediolateral ALT (mIALT), contains some GABAergic PNs; in the Drosophila brain, the great majority of ventral PNs (vPNs) are GABAergic and project through this tract to the lateral horn $(\mathrm{LH})$. Most excitatory PNs (ePNs), project through the medial ALT (mALT) to the mushroom body (MB) and the LH. Recent studies have shown that GABAergic vPNs play inhibitory roles at their axon terminals in the LH. However, little is known about the properties and functions of vPNs at their dendritic branches in the AL. Here, we used optogenetic and patch clamp techniques to investigate the functional roles of vPNs in the AL. Surprisingly, our results show that specific activation of vPNs reliably elicits strong excitatory postsynaptic potentials (EPSPs) in ePNs. Moreover, the connections between vPNs and ePNs are mediated by direct chemical synapses. Neither pulses of GABA, nor pharmagological, or genetic blockade of GABAergic transmission gave results consistent with the involvement of GABA in vPN-ePN excitatory transmission. These unexpected results suggest new roles for the vPN population in olfactory information processing. Keywords: olfaction, Drosophila, antennal lobe, electrophysiology, electrical synapses, chemical synapses, GABA,
lateral excitation

\section{INTRODUCTION}

The insect antennal lobe (AL) is a useful model system to study neural computations. Drosophila has been a particularly beneficial model system because it offers numerous genetic tools for labeling and manipulating the activity of neurons. In the Drosophila olfactory system, olfactory receptor neurons (ORNs) on peripheral appendages detect odorants and transfer this information to the AL. There, axon terminals of ORNs synapse onto projection neurons (PNs) including the ePNs (Liang and Luo, 2010; Rytz et al., 2013), which then transmit odor information to the mushroom body $(\mathrm{MB})$ and the lateral horn (LH) through the medial antennal lobe tract (mALT, Figure 1A). Each ORN expresses a single type of olfactory receptor (Vosshall et al., 2000) together with a non-odorant binding coreceptor (Orco, also known as Or83b, Larsson et al., 2004; Neuhaus et al., 2005). ORNs expressing the same receptor type project to the same glomerulus in the AL (Couto et al., 2005). Each ePN also projects its dendrites to a single glomerulus (Wong et al., 2002). Thus the identity of each ORN and PN can be defined by the glomerulus they target. ePNs have been studied extensively 

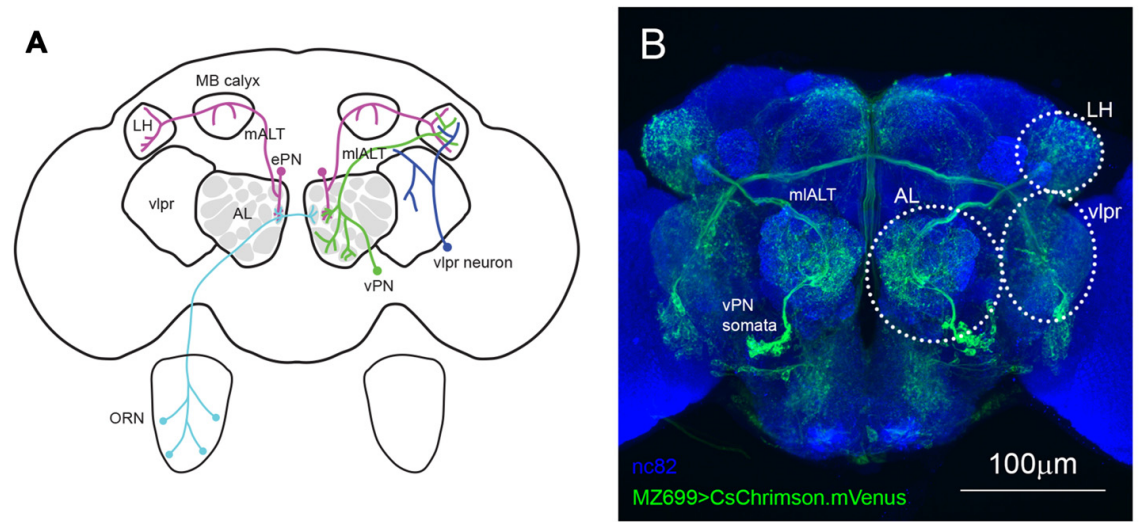

C
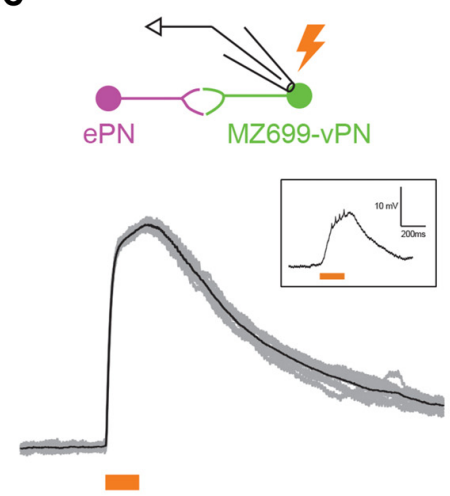

E

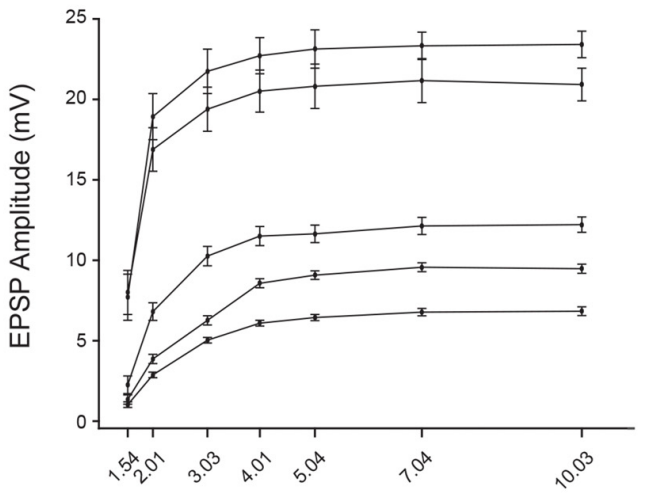

590 nm light intensity (\% of maximum)
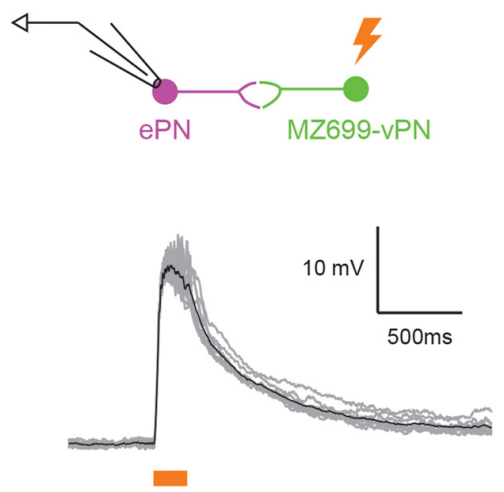

D

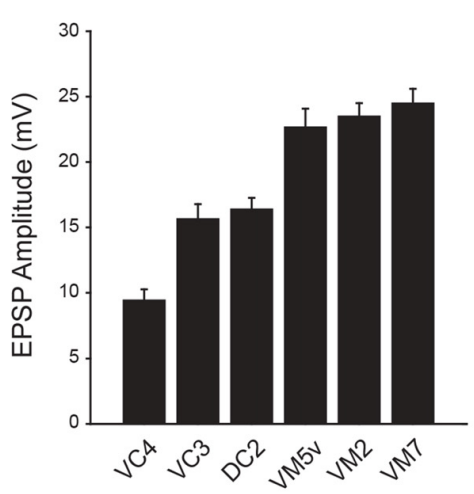

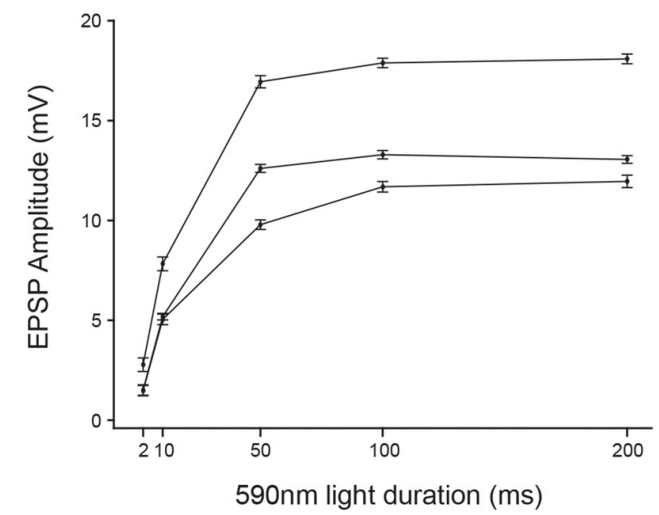

FIGURE 1 | MZ699+ neurons send glomerulus-specific excitation to excitatory projection neurons (ePNs). (A) Schematic representation of the Drosophila olfactory system. Two large neural populations in different brain areas, ventral PNs (vPNs; green) and ventrolateral protocerebrum (vlpr) neurons (blue) express MZ699-Gal4. (B) A confocal stack image of CsChrimson.mVenus expression driven by MZ699-Gal4. The neuropils were visualized by staining with nc82 antibody (blue). (C) Optogenetic activation of MZ699-Gal4 neurons with $\sim 64 \mu \mathrm{W} / \mathrm{mm}^{2} 200 \mathrm{~ms}$ whole-field $590 \mathrm{~nm}$ light elicited large depolarizations well above the spiking threshold in a MZ699 vPN (left); the average (black) of 10 trials (gray) from an example MZ699-vPN. The spikes the recorded neuron produced were small and difficult to see in the raw traces. A raw voltage trace of the same neuron upon stimulation with $\sim 1.3 \mu \mathrm{W} / \mathrm{mm}^{2}$ light for $200 \mathrm{~ms}$ is shown in the inset. A stronger light stimulus ( $\sim 64 \mu \mathrm{W} / \mathrm{mm}^{2} 200 \mathrm{~ms}$ whole-field $590 \mathrm{~nm}$ light) delivered to the brain elicited large and reliable excitatory postsynaptic potentials (EPSPs) in the recorded ePNs (right); the average (black) of 10 trials (gray) from an example ePN in VM5v glomerulus is shown. (D) The magnitude of excitation in ePNs upon MZ699-Gal4 > CsChrimson neurons was glomerulus-specific (mean \pm SEM, $n=4$ for VC4, $n=5$ for VC3, $n=3$ for DC2, $n=4$ for VM5V, $n=4$ for VM2, $n=2$ for VM7). Recorded ePNs were filled with dye, and their glomeruli were identified by comparing their positions with a standard atlas (Yu et al., 2010). (E) The magnitude of excitation in MZ699-Gal4 > CsChrimson ePNs varies with the intensity and duration of the light stimulus. The glomerular identities of the recorded ePNs are as follows: from the ePN with largest amplitude at 10.03\%, VM5V, VM3, DC2, VA1d and DM6, and from the ePN with largest amplitude at 200 ms, VA1d, VC4 and VC3. 
because they comprise the largest excitatory neural population in the Drosophila AL that sends information to other brain areas (Wilson, 2013).

Here we focus on a less studied population of PNs, the $\sim 50$ vPNs, which can send uniglomerular, multiglomerular or pan-AL dendritic projections (Figure 1A, Lai et al., 2008). MZ699-Gal4 is expressed in about $90 \%$ of all vPNs, which can be uniglomerular or multiglomerular (Figure 1B, MZ699-vPNs hereafter, Lai et al., 2008). Of these MZ699-vPNs, about $80 \%$ have been shown to be GABAergic by in situ hybridization against Gad1 (Okada et al., 2009); it is not known which neurotransmitter is expressed by the remaining $20 \%$ of MZ699vPNs. MZ699-vPNs project through the mediolateral antennal lobe tract (mlALT) only to the $\mathrm{LH}$ and are presumed to inhibit follower neurons. Indeed, the axon terminals of these MZ699-vPNs have been reported to inhibit their postsynaptic LH neurons (LHNs) through GABAergic synaptic transmission (Liang et al., 2013). On the other hand, very little is known about the functional roles dendritic arbors of vPNs play in the AL except that they have been reported to weakly excite ePNs through electrical synapses (Wang et al., 2014).

To better understand this excitatory transmission, we combined whole-cell patch clamp recordings with pharmacological and optogenetic manipulations to characterize connections from MZ699-vPNs onto ePNs. Surprisingly, our results show that MZ699-vPNs strongly, reliably, and monosynaptically excite the ePNs through chemical synapses. Our results suggest novel information processing roles for the second largest group of olfactory PNs in the AL.

\section{MATERIALS AND METHODS}

\section{Fly Strains}

Fly stocks were maintained on standard Drosophila medium at $\sim 25^{\circ} \mathrm{C}$ under a $12 \mathrm{~h} \mathrm{light/dark}$ cycle. The fly strains we used are as follows: MZ699-Gal4 (gift from T. Lee); 20xUAS-IVSCsChrimson.mVenus attP2 (Bloomington \#55136); Gad1 ${ }^{\text {MI09277 }}$ LexA::QFAD (gift from B.H. White); LexO-FLP (gift from K. Basler), tubP(FRT.stop)Gal80 (Bloomington \#38878), UASGad1-RNAi (VDRC \#32344), GH146-QF, QUAS-mCD8::GFP (Bloomington \#30038), UAS-Dcr2 (Bloomington, \#24646), 20xUAS-IVS-FRT.stop-spGFP1-10::CD4::HA, shakB ${ }^{2}$ (gift from C.J.H. Elliott), UAS-2HA.ort (gift from C.H. Lee). The genotypes of experiments in this study and brief descriptions of each genotype are as follows:

- Figures 1, 2A,B,C,D,E1,E2,F (control), G (control), Figures $\quad 3, \quad 4 \mathbf{A}, \mathbf{B}, \mathbf{E}, \mathbf{F}: \quad y w / w ; M Z 699-G a l 4 / 20 x U A S-I V S-$ CsChrimson.mVenus attP2 (Red shifted channel rhodopsin (CsChrimson) was expressed in MZ699-Gal4 positive cells).

- Figures 2E3,E4,F (experimental), G (experimental): yw/w;LexO-FLP/tubP(FRT.stop)Gal80;MZ699-Gal4, 20xUAS-IVS-CsChrimson.mVenus attP2/Gad1 ${ }^{\text {MI09277 }}$ LexA::QFAD (MZ699-Gal4 activity was suppressed by Gal80 only in Gad1 expressing cells, and expression of CsChrimson was lost in MZ699-vPNs as a result).
- Figures 4C (RNAi), D (RNAi): w, UAS-dicer2/w;;MZ699Gal4, 20xUAS-IVS-CsChrimson.mVenus attP2/UAS-Gad1RNAi (MZ699-Gal4 drove the expression of CsChrimson, double strand RNA against Gad1 and Dicer2. Dicer2 was expressed to enhance the efficiency of RNAi knock down).

- Figures 4C (control), D (control): w, UAS-dicer2/w;;MZ699Gal4, 20xUAS-IVS-CsChrimson.mVenus attP2/20xUASIVS-FRT.stop-spGFP1-10::CD4::HA (As a control for sequestration of Gal4 molecules, UAS-Gad1$R N A i$ was substituted with 20xUAS-IVS-FRT.stopspGFP1-10::CD4::HA).

- Figure 5 (Control): $y w /+; ; G H 146-Q F, Q U A S-m C D 8:: G F P /+$ (GH146-QF expressed mCD8::GFP marker in about two-thirds of ePNs).

- Figure 5 (shakB): shakB $B^{2}$ or shakB $B^{2} ; G H 146-Q F$, QUAS$m C D 8:: G F P /+$ (homozygotes of shakB $B^{2}$ mutation, which abolishes electrical synapses between excitatory local neurons (eLNs) and ePNs).

- Figure 6: $y w$;UAS-2HA.ort/+;MZ699-Gal4/GH146-QF, QUAS-mCD8::GFP (MZ699-Gal4 drove expression of histamine gated chloride channels (ort). ePNs were labeled with mCD8::GFP driven by GH146-QF).

\section{Whole-Cell Patch Clamp Recordings}

Patch-clamp electrodes (5-10 M $\Omega$ ) were pulled from borosilicate glass pipettes (Sutter Instrument) and filled with the following internal solution: (in $\mathrm{mM}$ ); 125 potassium aspartate, 10 HEPES, 4 MgATP, $0.5 \mathrm{Na} 3 \mathrm{GTP}, 1 \mathrm{EGTA}, 1 \mathrm{KCl}$, $\mathrm{pH}$ adjusted to $\sim 7.3$ with $\mathrm{KOH}$. To morphologically characterize the recorded cells, in most recordings $\sim 6 \mathrm{mM}$ biocytin hydrazide (Thermo Fisher Scientific, for ex vivo recordings) or $\sim 12 \mathrm{mM}$ neurobiotin (Vector Laboratories, for in vivo recordings) was added to the internal solution to label recorded cells. The osmolarity of the final internal solution was adjusted $265-280 \mathrm{mOsm} / \mathrm{kg}$ by adding potassium aspartate. For ex vivo recordings, brains of $\geqq 2$ day old flies were excised from the head capsule in extracellular saline and the perineural sheath above the ePN somata was removed with fine forceps. For in vivo recordings, the dorsal side of $\geqq 2$ day old female flies was restrained with gel epoxy on a plastic film with a small window over the fly's head. The dorsal side of the fly was immersed in extracellular saline and the dorsal cuticle between the compound eyes was removed. Both the antennae and maxillary palps were kept dry over the course of experiments. Fat tissue and tracheae over the ALs were removed and the muscle running along the esophagus was cut at the anterior side of the brain. The perineural sheath over the ePN somata was removed with fine forceps. The composition of the extracellular saline was as follows: $103 \mathrm{mM} \mathrm{NaCl}, 3 \mathrm{mM} \mathrm{KCl}$, $5 \mathrm{mM} \mathrm{N}$-[Tris(hydroxymethyl)methyl]-2-aminoethanesulfonic acid, $10 \mathrm{mM}$ trehalose, $10 \mathrm{mM}$ glucose, $26 \mathrm{mM} \mathrm{NaHCO}$, $1 \mathrm{mM} \mathrm{NaH}_{2} \mathrm{PO}_{4}, 1.5 \mathrm{mM} \mathrm{CaCl}_{2}$, and $4 \mathrm{mM} \mathrm{MgCl}_{2}$. $\mathrm{pH}$ was equilibrated around 7.3 by bubbling with $95 \% \mathrm{O}_{2} / 5 \% \mathrm{CO}_{2}$. The osmolarity of the external solution was $\sim 280 \mathrm{mOsm} / \mathrm{kg}$ without adjustment. The flow rate of perfusion was set at $2 \mathrm{~mL} / \mathrm{min}$ by a RP-1 peristaltic pump (RAININ) and the saline temperature was maintained at $23-24^{\circ} \mathrm{C}$ by TC-324B, SH-27B 
A
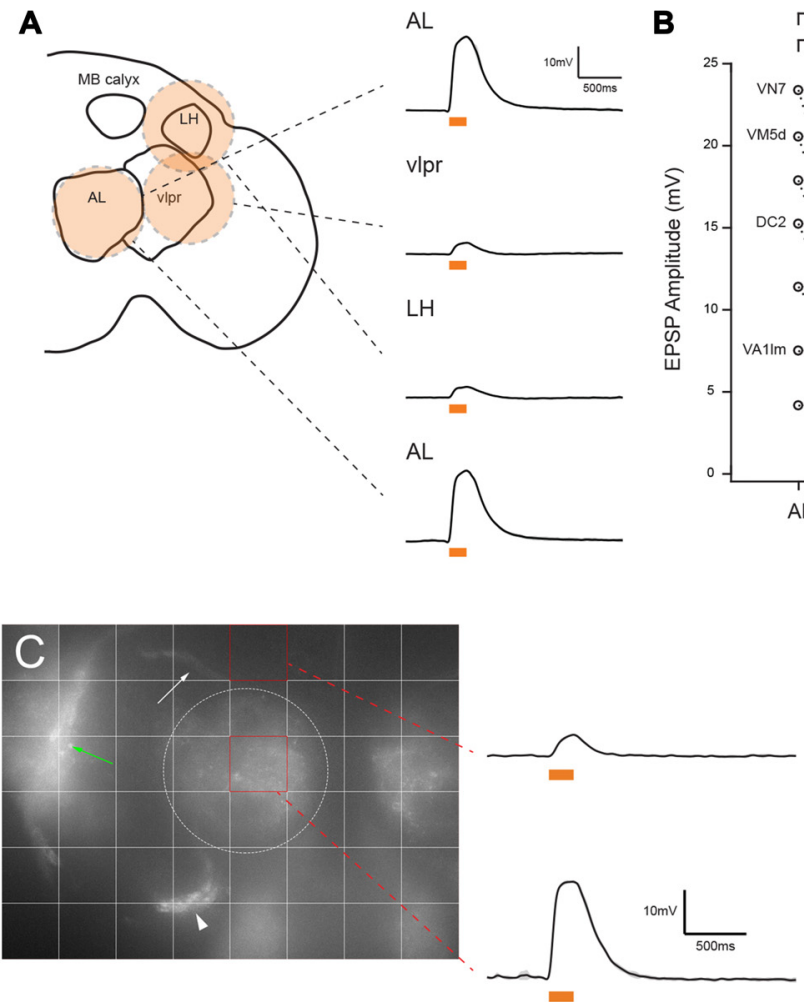

B

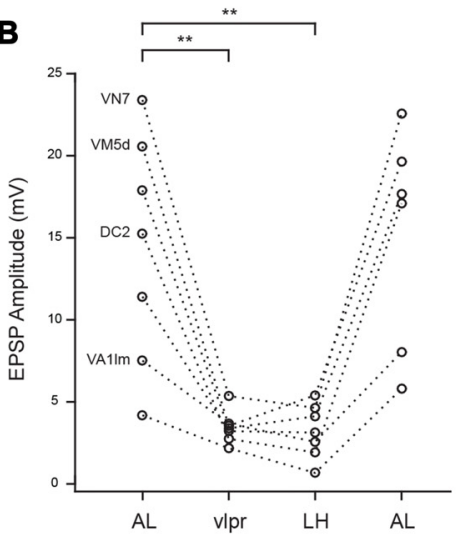

D

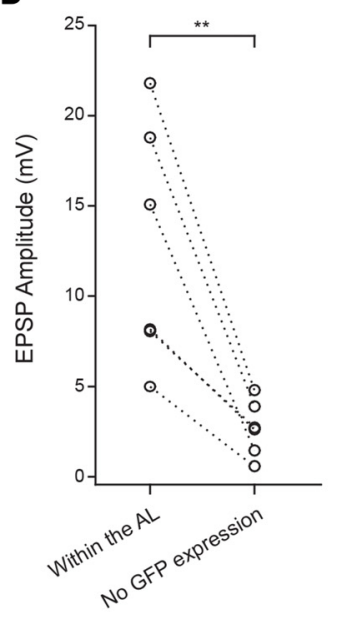

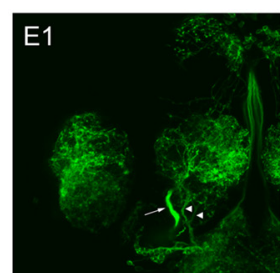

E3
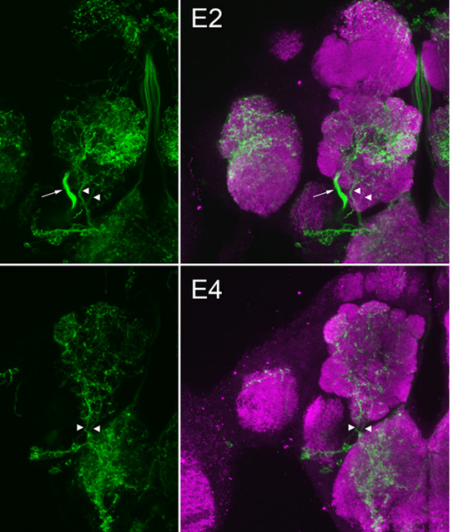

$\mathbf{F}$
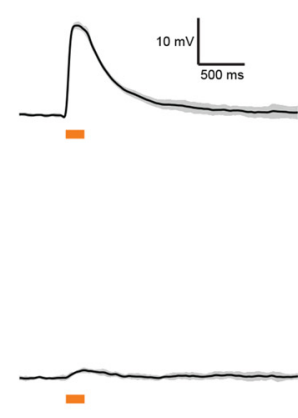

G

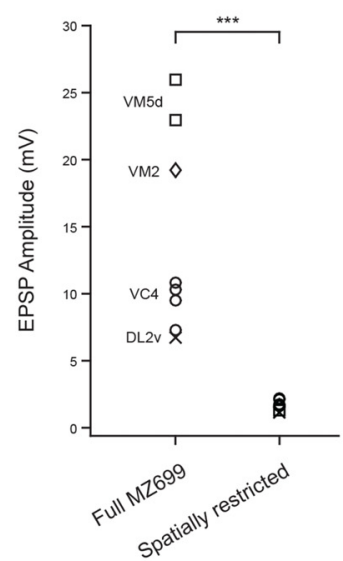

FIGURE 2 | MZ699-vPNs are the source of excitation to the ePNs. (A) Schematic illustration shows how a circular flash of $590 \mathrm{~nm}$ light was directed sequentially to the antennal lobe (AL), lateral horn (LH), vlpr and then back to the AL. Example traces from a VM7 ePN are shown on the right (mean \pm SD). (B) All recorded ePNs showed the greatest depolarization when $590 \mathrm{~nm}$ spot was directed to the $\mathrm{AL}\left(n=7\right.$, paired $t$-test, $\left.{ }^{* *} p<0.01\right)$. The identities of four ePNs filled with biocytin hydrazide are shown on the left. (C) Grid stimulation of areas devoid of CsChrimson expression (e.g., upper red square) induced much smaller depolarization in an ePN (mean \pm SD) than stimulation of areas including the AL (e.g., lower red square). The bundle of MZ699-vPNs 


\section{FIGURE 2 | Continued}

projecting to the LH, the MZ699-vPNs' somata, and the vipr neurons are indicated with a white arrow, a white arrowhead and a green arrow, respectively. The AL is encircled with a white dotted line. (D) In all six ePNs, stimulation of an area without CsChrimson expression induced smaller depolarization than stimulation of areas including the $\operatorname{AL}\left({ }^{* *} p<0.01\right.$, paired $t$-test). The glomerular identities of recorded ePNs are as follows: from the ePN with the largest magnitude with stimulation of AL, VM7, VM2, VM2, DC2, DC2 and VA1d. (E1,E2) MZ699-Ga/4 drove expression of CsChrimson (green) in VPNs (an axon bundle indicated with an arrow) and two fibers innervating the AL (arrowheads). (E3,E4) A genetic intersection strategy (crossing MZ699-Ga/4 and Gad1-LexA::QFAD) eliminated expression of CsChrimson selectively in the vPNs while expressing CsChrimson specifically in the two fibers (arrowheads). (F) Example voltage traces from two ePNs in the same type of glomerulus (VM5d). Top: full MZ699-Gal4; Bottom: spatially restricted genotype (mean \pm SD). (G) Compared to results from the full MZ699-Gal4 group, depolarization magnitude was much smaller when expression of CsChrimson in vPNs was eliminated from MZ699-Ga/4 expression pattern ( $n=8$ for control group, $n=7,1.68 \pm 0.14 \mathrm{mV}$ for experimental group,

${ }^{* * *} p<0.001$, two-way analysis of variance (ANOVA)). This result shows that, of the neuron populations tested, only MZ699-VPNs provide excitation to the ePNs.

and TS-70B inline heater and thermistor (Warner Instruments). The electrode was directed to the anterodorsal ePN soma under visual guidance using an Axio Examiner.A1 (Zeiss) microscope with a $\times 40$ water-immersion objective. ePNs were identified by the location of their somata and their characteristically small spike size $(\sim 10 \mathrm{mV})$. Recordings were acquired with a MultiClamp 700B (Molecular Devices) amplifier and signals were low-pass filtered at $10 \mathrm{kHz}$ and digitized at $20 \mathrm{kHz}$ by a Digidata 1440B (Molecular Devices) digitizer. The resting membrane potential was kept $\sim-58 \mathrm{mV}$ by injecting a small hyperpolarizing current in current clamp mode. Voltages were not corrected for the liquid junction potential.

\section{Optogenetics}

Flies were cultured on standard fly food covered with potato flakes rehydrated with $1.75 \mathrm{mM}$ all trans-Retinal (ATR). ATR was prepared as $35 \mathrm{mM}$ in ethanol as a stock and diluted for use 20-fold. For flashes of whole-field illumination of $590 \mathrm{~nm}$ light, the shutter on the Colibri LED light source was controlled using the Micro Toolbox (Zeiss). For localized stimulation of $590 \mathrm{~nm}$ light on the AL, ventrolateral protocerebrum (vlpr) and LH, illumination patterns generated in PolyScan2 or PolyLite software (Mightex) were flashed on the brains using the BioLED Light Source Control Module and Polygon400 Dynamic spatial illuminator (Mightex). Both the shutter of Colibri and BioLED Light Source Control Module were triggered with TTL input from a Master-8 (A.M.P.I) timer, which was synchronized with data acquisition by the Digidata1440B.

\section{Pharmacology}

TTX, PTX, $\mathrm{CdCl}_{2}$ and histamine were prepared as $2 \mathrm{mM}$, $5 \mathrm{mM}, 100 \mathrm{mM}$ and $100 \mathrm{mM}$ stocks in distilled water and diluted at 1:2000, 1:500, 1:1000 and 1:1000, respectively, for use. CGP54626 was prepared as a $50 \mathrm{mM}$ stock in DMSO and diluted at 1:1000. Drug solutions were perfused for about $5 \mathrm{~min}(\sim 10 \mathrm{~mL})$ before their effects were tested. To confirm that genetically expressed His-Cl channels effectively suppress MZ699-Gal4 expressing neurons, we expressed both His-Cl channels and CsChrimson in MZ699-Gal4 neurons. Strong EPSPs evoked in ePNs were effectively reduced in the presence of $100 \mu \mathrm{M}$ histamine (data not shown).

\section{Odor Stimulation}

Benzaldehyde, 2-Octanone, Pentyl acetate, Ethyl acetate and Ethyl butyrate were diluted at $0.3 \% \mathrm{v} / \mathrm{v}$ with mineral oil in glass vials. A vial with only mineral oil and an empty vial were included as negative controls. Two hundred $\mathrm{mL} / \mathrm{min}$ air flow filtered through activated charcoal was directed to the fly through a $3 \mathrm{~mm}$ i.d. tube whose outlet was set $8 \mathrm{~mm}$ away from the fly. One hundred $\mathrm{mL} / \mathrm{min}$ air flow was pulsed by a PV820 pneumatic Picopump (World Precision Instruments), which was triggered by a Master-8 (A.M.P.I) timer, filtered through activated charcoal, and passed through an odor bottle. Tubes delivering the odorized air were merged into the constant flow $16 \mathrm{~cm}$ away from the outlet of the tube delivering the constant flow to the antennae. The $100 \mathrm{~mL} / \mathrm{min}$ air flow was manually switched with a custom-made olfactometer and directed to one of seven vials. Odor pulse duration was $1 \mathrm{~s}$ and pulses were presented at $40 \mathrm{~s}$ intervals. For recordings in the antennal-less preparation, the odors ethyl acetate and ethyl butyrate, which typically induce strong lateral excitation in antennal ePNs, were mainly used. For recordings in preparations with intact antennae and palps, the five odors listed above were used to pre-test preparations before recordings began, and odors that elicited an increase in a spike rate were then used for the following recordings in the absence or presence of histamine.

\section{Immunohistochemistry}

Brains used for recordings were fixed in $4 \%$ paraformaldehyde in Sorenson's buffer (0.2 M, pH 7.2) for $30 \mathrm{~min}$ at room temperature. Brains were washed in Sorenson's buffer, incubated in $10 \%$ goat serum in $0.3 \%$ Triton X-100 in Sorenson's buffer (PBT) for $1 \mathrm{~h}$ at room temperature, and then incubated in 1:5 or 1:10 anti-Bruchpilot antibody (nc82, Developmental Studies Hybridoma Bank) and $1 \%$ goat serum in PBT for 2 days at $4^{\circ} \mathrm{C}$. Brains were washed in PBT for $10 \mathrm{~min}$ at room temperature four times and then incubated in PBT containing 1:500 rabbit antiGFP-Alexa488 (catalog \#A-21311, ThermoFisher Scientific), 1:100 or 1:200 goat anti-mouse Alexa Fluor 568 (catalog \#A11031), $500 \mu \mathrm{g} / \mathrm{L}$ streptavidin Alexa Fluor 633 (catalog \#S21375) and $1 \%$ goat serum for 2 days at $4^{\circ} \mathrm{C}$. After washing in PBT for $10 \mathrm{~min}$ at room temperature four times, brains were mounted on a glass slide with $70 \%$ glycerol in Sorenson's buffer.

\section{Data Analysis}

Analyses were performed using custom programs written in MATLAB (MathWorks). To measure EPSP amplitude and effects of GABA, voltage traces were low-pass filtered at $13 \mathrm{~Hz}$ to remove spikes. The baseline membrane potential (1 s period prior to the light stimulus or GABA pulse) was then subtracted from the whole trace to compute deflections from the baseline. To compute EPSP latency from the light onset a second order 
parabolic function was fit to the EPSP, and EPSP onset was defined as the time this function intersected with the baseline (voltage recorded during a $1 \mathrm{~ms}$ period before the light stimulus). Peri-stimulus time histograms (PSTHs) were calculated to characterize odor-elicited spiking responses in vivo. Spikes trains for each trial were binned ( $50 \mathrm{~ms}$ bins overlapping by $25 \mathrm{~ms}$ ), and then, for each trial, the average spike rate during a $2 \mathrm{~s}$ period prior to the odor stimuli was subtracted from each bin. Bins were then averaged across trials to yield an average PSTH. From this, the maximum spike rate was calculated from the bin with the highest count, and the total spike count was computed as the area under the curve of the PSTH. Multiple comparisons were corrected with Bonferroni's method.

\section{RESULTS}

\section{vPNs Strongly and Reliably Excite ePNs}

MZ699-Gal4 is expressed in about $90 \%$ of vPNs, about $80 \%$ of which are GABAergic (Lai et al., 2008; Okada et al., 2009). It is not known which neurotransmitter the remaining $20 \%$ of MZ699-vPNs express. To characterize connections from vPNs to ePNs we used an optogenetic technique: we expressed CsChrimson driven by MZ699-Gal4 in vPNs, and activated them with $200 \mathrm{~ms}$ of whole-field $590 \mathrm{~nm}$ light ( 64 $\mu \mathrm{W} / \mathrm{mm}^{2}$ corresponding to $5 \%$ intensity of our whole-field light stimulation) while performing whole-cell patch clamp recording from ePNs. This light stimulus induced huge depolarizations in a MZ699-vPN well above the spiking threshold (Figure 1C). Because the great majority of vPNs are GABAergic we predicted that activating them as a population would inhibit the ePNs. Surprisingly, vPN activation reliably excited ePNs (Figure 1C); when we activated vPNs this way, we never observed inhibition in an ePN. The magnitude of excitation was generally much stronger than would be expected from an electrical synapse (Wang et al., 2014) and varied by glomerulus (Figure 1D). The magnitudes of these EPSPs were light-dose dependent and saturated at around 5\% of the maximum intensity of our whole-field light source. A $200 \mathrm{~ms}$ stimulus sufficed to induce maximal EPSPs (Figure 1E). Whole-field $200 \mathrm{~ms} 590 \mathrm{~nm}$ light did not excite ePNs in animals of the same genotype that were not supplemented with ATR $(1.05 \pm 0.29 \mathrm{mV}$ of excitation, $n=5)$.

We sought to localize the source of excitatory input to the ePNs. MZ699-Gal4 (and thus CsChrimson) is also expressed in a second large group of cells, the vlpr neurons, located in the vlpr (Figures 1A,B). These neurons may make direct contact with axon terminals of ePNs in the LH (Figure 1B), and could therefore be a source of the light-driven excitatory input to the ePNs. To test this possibility, we used a digital mirror device (DMD) to selectively illuminate the preparation, directing a narrow circle of $590 \mathrm{~nm}$ light centered on the $\mathrm{AL}, \mathrm{LH}$ or vlpr (Figure 2A). The magnitude of excitation observed when we illuminated the AL was significantly much larger than when we illuminated the LH or vlpr (Figure 2B), indicating that the source of the EPSPs in the ePNs upon activation of MZ699Gal4 positive neurons is located in the AL. We more precisely localized the source of excitation to ePNs by directing narrower beams of light to the brain. In all six ePNs recorded, illuminating within the AL induced the largest EPSPs, while illuminating areas devoid of MZ699-Gal4 expression induced significantly smaller EPSPs (Figures 2C,D, $21.67 \pm 4.14 \%$ of the peak amplitude in the AL, $n=6$ ). Close examination of the MZ699Gal4 expression pattern in the AL revealed two labeled fibers innervating the AL in addition to vPN neurites (Figures 2E1,E2). To test if these additional fibers are the source of excitation to the ePNs, we used an intersectional genetic approach (MZ699Gal4 and Gad1-LexA::QFAD2) so that activating expression of Gal80 resulted in suppressing expression of CsChrimson only in vPNs (Figures 2E3,E4). When we activated the two additional fibers with light flashes, we found that the magnitude of excitation in ePNs was significantly lower than that on activation of MZ699-Gal4 positive neurons in corresponding glomeruli (Figures 2F,G). Together, these results demonstrate that vPNs excite the ePNs in the AL, probably through dendrodendritic connections.

\section{vPNs Are Monosynaptically Connected to ePNs through Chemical Synapses}

vPNs have previously been shown to evoke small excitatory potentials in ePNs through bidirectional electrical synapses composed of shakB containing gap junctions (Wang et al., 2014). To further characterize vPN-ePN connections, we optogenetically activated CsChrimson-expressing vPNs with brief (100\% wide field $590 \mathrm{~nm}$ light, $2 \mathrm{~ms}$ ) flashes of light and measured the latency from the onset of the light flashes to the onset of EPSPs in ePNs. This method of measuring the latency from light onset to onset of EPSCs to establish monosynaptic connectivity from channel rhodopsin (ChR) expressing cells to recorded cells is well-established (Kohara et al., 2013; Root et al., 2014). All ePNs examined this way showed EPSPs of short latency $(n=5,1.33 \mathrm{~ms} \pm 0.13 \mathrm{~ms})$, consistent with monosynaptic connectivity (Figures 3A,B). These short latency EPSPs could comprise both fast potentials through electrical synapses followed by slower chemical polysynaptic potentials from unknown neurons. To test this possibility, we blocked chemical synaptic release by bathing the preparation with $\mathrm{Cd}^{2+}$. By subtracting the residual $\mathrm{Cd}^{2+}$-insensitive voltage traces from the traces recorded before drug application we could reveal any purely chemical transmission component. We found the response latency of the remaining chemical component still had a short latency (1.27 ms $\pm 0.10 \mathrm{~ms}, n=5$, Figures $\mathbf{3 A}, \mathbf{B})$. These results rule out the possibility that electrical synapses alone underlie fast EPSPs from MZ699-vPNs to ePNs, and are consistent with ePNs receiving monosynaptic chemical excitation from the vPNs.

To further test whether these connections were monosynaptic, we blocked polysynaptic transmission by bathing the preparation with TTX. Blockade of voltage-gated $\mathrm{Na}^{+}$ channels has been used previously to establish monosynaptic connections from $\mathrm{ChR}$ expressing cells to recorded cells (Franks et al., 2011; Sugimura et al., 2016). We found that EPSPs in ePNs elicited by optogenetic activation of vPNs 

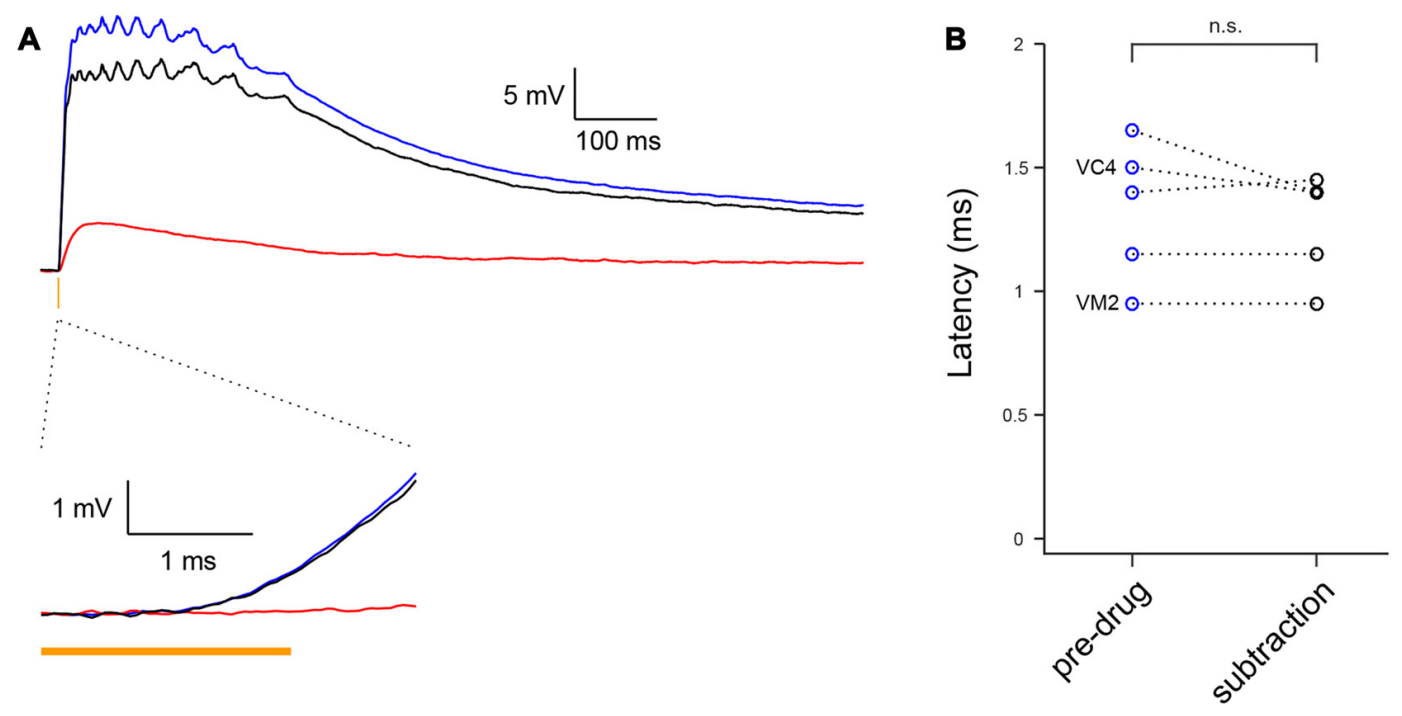

C

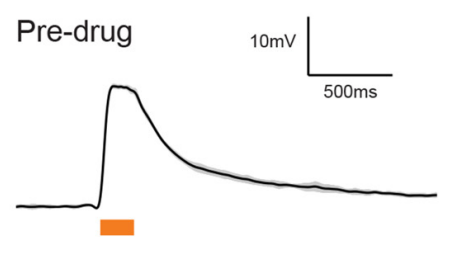

$1 \mu \mathrm{M}$ TTX

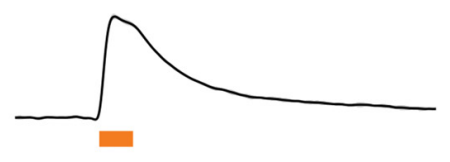

$1 \mu \mathrm{M}$ TTX

$100 \mu \mathrm{M} \mathrm{CdCl}_{2}$

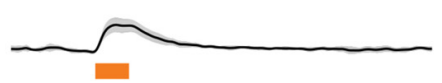

D

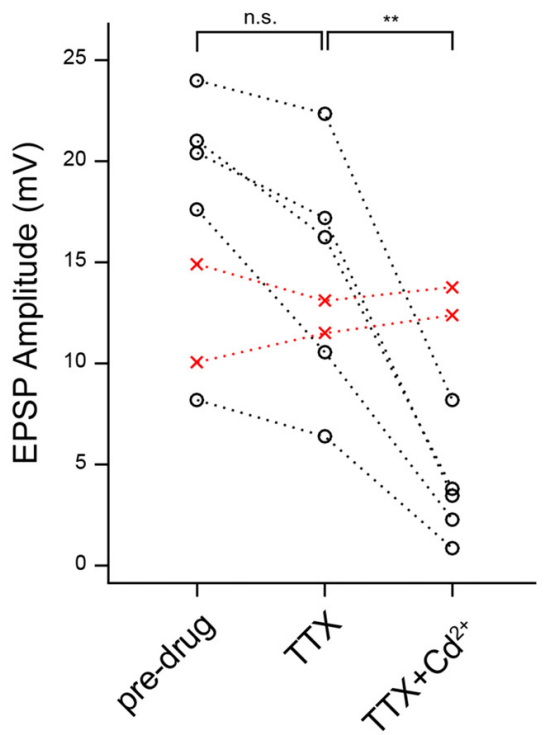

$\mathbf{E}$

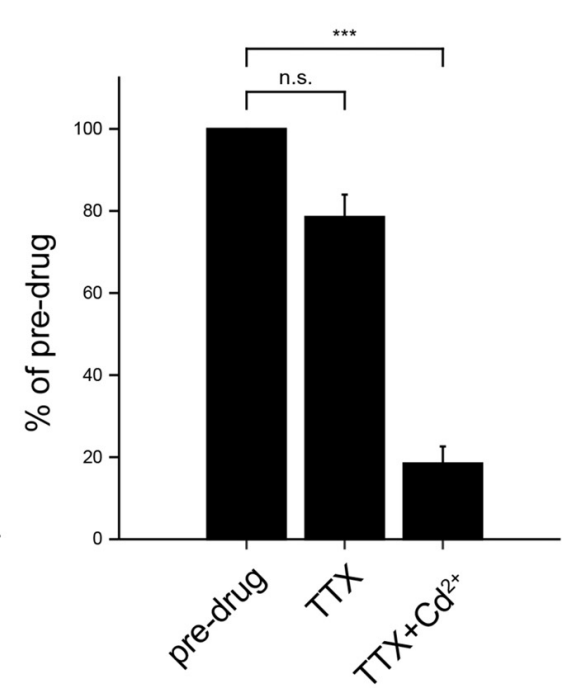

FIGURE 3 | MZ699-vPNs monosynaptically excite ePNs through chemical synapses. (A) Average voltage traces of ePN depolarization on optogenetic activation of MZ699-Ga/4+ neurons with $2 \mathrm{~ms} 590 \mathrm{~nm}$ pulse before (blue) and after perfusion with $\mathrm{Cd}^{2+}$ to block chemical transmission (red); the subtraction trace (black) reveals the chemical synaptic transmission component of the response. (B) The EPSPs latency from the light onset was very brief, consistent with a monosynaptic chemical connection $\left(n=5,1.33 \pm 0.13 \mathrm{~ms}\right.$ before $\mathrm{Cd}^{2+}, 1.27 \pm 0.10 \mathrm{~ms}$ after $\left.\mathrm{Cd}^{2+}, p=0.32\right)$. The identities of two ePNs filled with biocytin hydrazide are shown on the left. (C) Example traces showing depolarization of an ePN induced by optogenetic activation of MZ699-Ga/4+ neurons before drug application (top), after infusion of $1 \mu \mathrm{M}$ tetrodotoxin (TTX; middle), and after subsequent infusion of $1 \mu \mathrm{M}$ TTX and $100 \mu \mathrm{M}$ Cd ${ }^{2+}$ (bottom). (D) Depolarization magnitude was slightly reduced with $1 \mu \mathrm{M} \operatorname{TX}\left(n=5, p=0.027\right.$, paired $t$-test) but greatly reduced with subsequent infusion of $1 \mu \mathrm{M} \Pi \mathrm{TX}$ and $100 \mu \mathrm{M} C d^{2+}(n=5$, ${ }^{* *} p<0.01$, paired $t$-test). Replacement of external saline with a control drug-free saline did not cause any change in EPSP amplitude (red crosses). The glomerular identities of the recorded ePNs are as follows: from the ePN with largest amplitude in pre-drug condition, VM3, VC3, D, DC2 and VA1d for the drug application group (black circle), and VC3 and VA2 for the drug-free saline group (red circles). (E) A bar graph shows the EPSP amplitude normalized by pre-drug responses $(n=5$, TTX: $78.54 \pm 5.45 \%, p=0.017, \pi \mathrm{TX}+\mathrm{Cd}^{2+}: 18.48 \pm 4.13 \%,{ }^{* * *} p<0.001$, paired $t$-test).

persisted even in the presence of TTX, though the EPSPs were slightly smaller in amplitude $(78.54 \pm 5.45 \%$ of pre-drug, $n=5, p=0.027$, Figures 3C-E). Because TTX blocks the generation of action potentials by voltage-gated $\mathrm{Na}^{+}$channels, these optogenetically induced EPSPs in ePNs were caused by CsChrimson-triggered synaptic release from vPN presynaptic 
sites. When TTX and $\mathrm{Cd}^{2+}$ were both added to the bath, residual EPSPs were greatly and significantly reduced $(18.48 \pm 4.13 \%$ of pre-drug, $n=5, p=0.0030$, Figures $3 \mathrm{C}-\mathrm{E}$ ), indicating that they were induced largely through chemical transmission. It is possible that local circuits (Berck et al., 2016) could mediate indirect connections between vPNs and ePNs; for example, optogenetically activated vPNs could excite Chrimson negative neurons which then excite ePNs in a spike-independent manner. However, our pharmacological manipulations combined with our measurements of very brief EPSP latencies make this scenario unlikely, and rather suggest that vPNs mainly excite ePNs through monosynaptic chemical synapses.

\section{GABA May Not Contribute to vPN-ePN Excitatory Transmission}

What transmission mechanism allows predominantly GABAergic vPNs to excite ePNs? GABA is a major inhibitory neurotransmitter in the Drosophila central nervous system; it causes inhibition by activating $\mathrm{GABA}_{\mathrm{A}}$ and $\mathrm{GABA}_{\mathrm{B}}$ receptors, leading to the influx of $\mathrm{Cl}^{-}$ions and efflux of $\mathrm{K}^{+}$ions, respectively (Sodickson and Bean, 1996; Hosie et al., 1997). GABA is also known to depolarize postsynaptic neurons in some circuits (notably in the developing mammalian brain) where elevated intracellular concentrations of $\mathrm{Cl}^{-}$shift the $\mathrm{Cl}^{-}$ reversal potential such that activating $\mathrm{GABA}_{\mathrm{A}}$ receptors leads to a depolarizing efflux of $\mathrm{Cl}^{-}$ions. However, we found that the amplitude of EPSPs triggered in ePNs by photoactivation of CsChrimson expressing MZ699-vPNs was not affected by bath application of $10 \mu \mathrm{M}$ PTX $(n=5, p=0.059$, Figures 4A,B), a treatment that effectively blocks $\mathrm{GABA}_{\mathrm{A}}$ receptors in Drosophila (Olsen and Wilson, 2008). This suggests vPN-triggered EPSPs in ePNs are not mediated by $\mathrm{GABA}_{\mathrm{A}}$ receptors. Further, our intracellular patch solution contained low concentrations of $\mathrm{Cl}^{-}$ion making it unlikely that the $\mathrm{Cl}^{-}$reversal potential of recorded neurons favored their depolarization by GABA (Raimondo et al., 2017). GABA has been shown to activate other types of receptors, potentially leading to excitation. For example, it has been reported that GRD (GABA and glycine-like receptor of Drosophila) and ligand-gated chloride channel homolog 3 (LCCH3) form GABA-gated heteromultimeric cation channels in the Xenopus laevis oocyte heterologous expression system. However, GABA-elicited inward flow of cations in oocytes expressing GRD and LCCH3 could also be blocked by application of $10 \mu \mathrm{M}$ PTX (Gisselmann et al., 2004), excluding the possibility that GRD and $\mathrm{LCCH} 3$ underlie the excitatory input from MZ699-vPNs to ePNs.

To test if GABA synthesis in the MZ699-vPNs is necessary for the depolarization of ePNs, we used RNAi to knock down the Gad1 gene in neurons expressing MZ699-Gal4. We then compared the amplitudes of EPSPs triggered in ePNs by photoactivation of CsChrimson expressing MZ699-vPNs between control and RNAi animals. Although we found that GABA immunostaining was significantly reduced in RNAi MZ699-vPNs (Figure 4C, $n=8$ hemispheres for control, $n=8$ hemispheres for RNAi, $p<0.001, t$-test), EPSP amplitude on photoactivation of MZ699-vPNs was not reduced in the nine glomeruli we tested (Figure 4D, $n=25$ for control, $n=21$ for RNAi, $p=0.59$, two-way ANOVA).

We further tested whether pressure ejecting GABA over the desheathed $\mathrm{AL}$ causes depolarizing responses in ePNs. In a normal saline bath, GABA pulses reliably hyperpolarized the ePNs, indicating that the GABA pulses were effectively delivered to the recorded cells (Figure $4 \mathrm{E}$, top). Since hyperpolarizing potentials mediated by $\mathrm{GABA}_{\mathrm{A}}$ and $\mathrm{GABA}_{\mathrm{B}}$ receptors could mask a possible excitatory response, we then pulsed GABA in the presence of $10 \mu \mathrm{M}$ PTX and $50 \mu \mathrm{M}$ CGP54626, a GABA receptor antagonist effective in Drosophila (Wilson and Laurent, 2005). In the presence of the antagonists, we never observed excitatory responses to GABA (Figure $4 \mathrm{E}$ bottom, Figure $4 \mathrm{~F}$, $n=5,-7.57 \pm 1.56 \mathrm{mV}$ before antagonists, $0.19 \pm 0.10 \mathrm{mV}$ after antagonists). All the above results suggest GABA does not mediate the excitatory transmission at $\mathrm{vPN}-\mathrm{ePN}$ synapses.

\section{vPN-ePN Excitatory Connections May Not Underlie Wide-Spread Lateral Excitation in the AL}

What is the functional significance of the direct vPN-ePN chemical synapses? We hypothesized that vPNs could contribute to lateral excitatory interactions among glomeruli because a subset of vPNs has multiglomerular branches in the AL and because MZ699-vPNs, as a group, strongly excite ePNs. In the Drosophila AL, odor-elicited lateral excitatory interactions among glomeruli persist in preparations in which both antennal nerves were acutely severed, and thus lack direct afferent inputs from the antennae to the antennal ePNs (Figure 5A). These lateral excitatory interactions have been reported to spread through shakB-dependent electrical synapses between ePNs and two or three krasavietz-Gal4 expressing excitatory LNs (eLNs; Yaksi and Wilson, 2010). To compare the possible roles of shakB-dependent electrical synapses and vPN-ePN excitatory connections, we tested odor-evoked lateral excitatory inputs to antennal ePNs in several glomeruli in shakB $B^{2}$ mutants, which lack the neural isoform of shakB innexin molecules (Zhang et al., 1999). Consistent with a previous report (Yaksi and Wilson, 2010), lateral inputs to antennal ePNs in two glomeruli, DA1 and VA5, were greatly reduced and appeared to be dominated by inhibition. This trend was observed in all other glomeruli in the same lateral ePN lineage as DA1 and VA5 (for ethyl acetate, peak depolarization was $5.71 \pm 1.89 \mathrm{mV}$ in control flies and $0.76 \pm 0.67 \mathrm{mV}$ in shakB ${ }^{2}$ mutants. For ethyl butyrate, peak depolarization was $6.23 \pm 2.60 \mathrm{mV}$ in control flies and $0.48 \pm 0.46 \mathrm{mV}$ in shakB ${ }^{2}$ mutants; $n=13$ for control and $n=6$ for shak $B^{2}$ mutant including ePNs in DA1 and VA5, $p<0.001$, Wilcoxon rank sum test). However, in two other glomeruli, VC3 and VM5v, in the same shakB $B^{2}$ mutants we observed large amplitude odor-elicited depolarizations (Figure 5B). These results indicate that shakBdependent electrical synapses between ePNs and eLNs do not provide the sole mechanism underlying lateral excitatory interactions in the Drosophila AL.

To test whether vPNs contribute to these lateral excitatory responses, we suppressed their activity by expressing shunting 


\section{A}

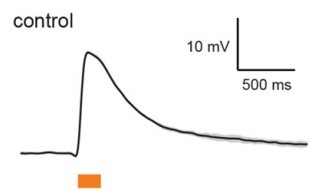

$10 \mu \mathrm{M}$ PTX

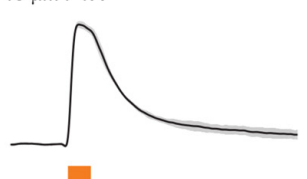

-
B

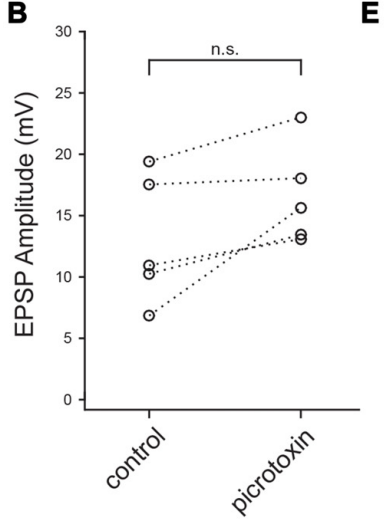

E

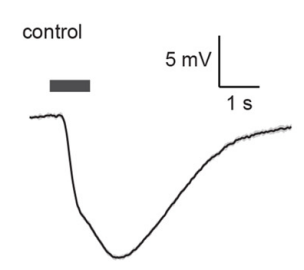

$10 \mu \mathrm{M}$ PTX $+50 \mu \mathrm{M}$ CGP54626

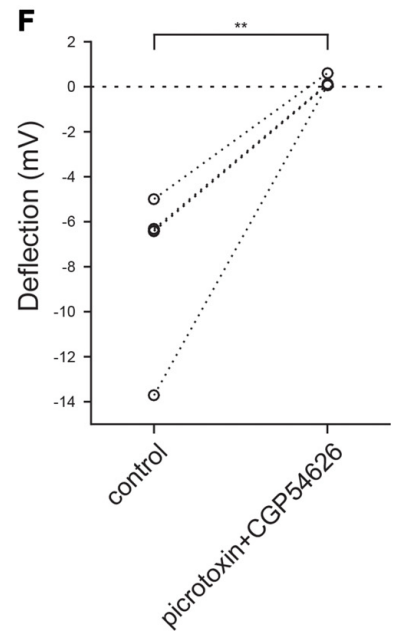

C
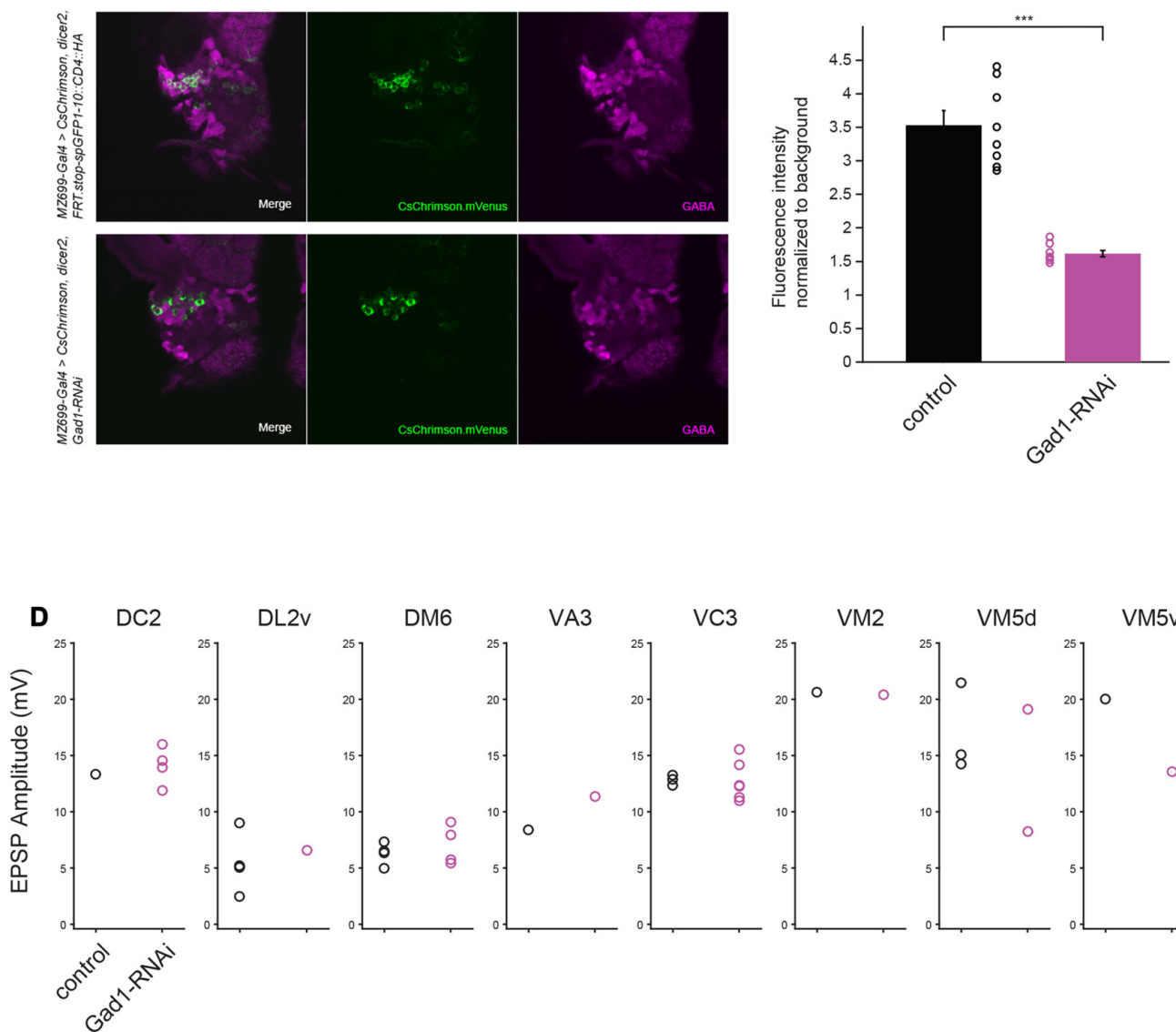

DM6

VA3

VC3
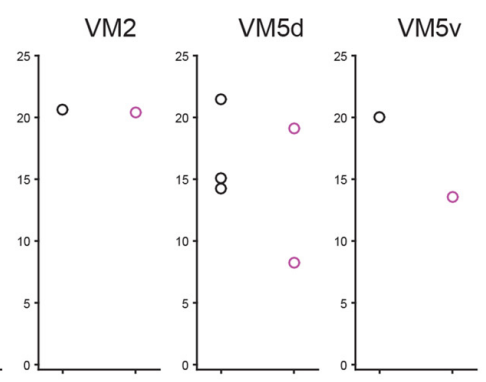

VM5v

VM7
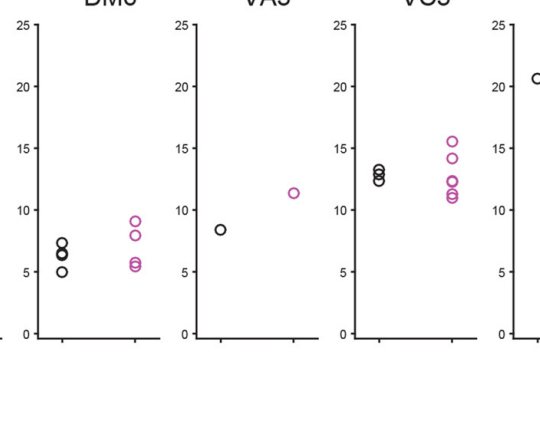

.

FIGURE 4 | GABA may not contribute to vPN-ePN excitatory synaptic connections. (A) Example traces show EPSPs in ePNs triggered by optogenetic activation of VPNs before (top) and after (bottom) application of GABA $A_{A}$ blocker $10 \mu \mathrm{M} \mathrm{PTX} \mathrm{(mean} \pm$ SD), both in the presence of $1 \mu \mathrm{M}$ TTX to block indirect activation of the neurons. (B) Perfusion with $10 \mu \mathrm{M}$ PTX did not change the depolarization magnitude in any of five experiments $(n=5, p=0.059$, paired $t$-test). The glomerular identities of recorded ePNs are as follows: from the ePN with largest amplitude in control condition, VM5v, VM3, VA1d, VC4 and VC4. (C) RNAi mediated knock-down of Gad1 with MZ699-Gal4 resulted in decreased expression of GABA in MZ699-vPNs. Decrease in GABA staining signal in the vPN somata was more than two-fold ( $n=8$ hemispheres for control and $n=8$ hemispheres for RNAi animals, ${ }^{* * *} p<0.001, t$-test). (D) Diminishing GABA expression by Gad1 knock-down driven by MZ699-Ga/4 did not change EPSP magnitude of ePNs in nine glomeruli upon optogenetic activation of MZ699-Ga/4+ neurons $(n=25$ for control: black circles, $n=21$ for RNAi: red circles, $p=0.59$, two-way ANOVA). (E) Example traces show that puffs of $1 \mathrm{mM}$ GABA delivered to the AL before (top) and after (bottom) bath application of $10 \mu \mathrm{M}$ PTX and $50 \mu \mathrm{M}$ CGP54626 (mean \pm SD), both in the presence of $1 \mu \mathrm{M}$ TTX, did not elicit depolarizing responses in ePNs; similar results obtained from five experiments are shown in (F) (The responses of three ePNs in the control group and four ePNs in experimental group were so similar that the plots for these cells are superimposed ${ }^{* *} p<0.01$, paired $t$-test). 


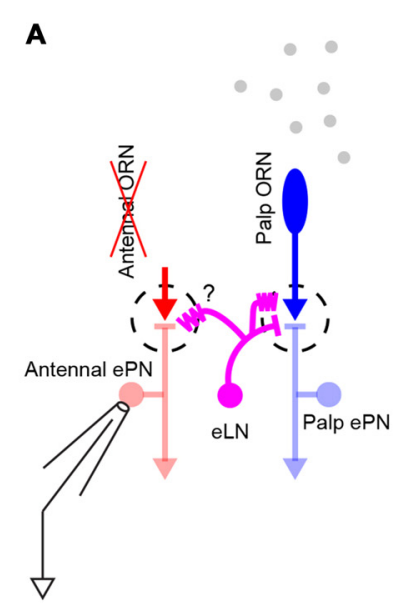

\section{B

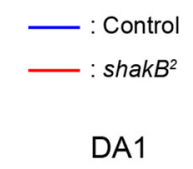

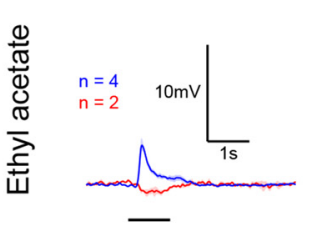

VA5

VC3

VM5d
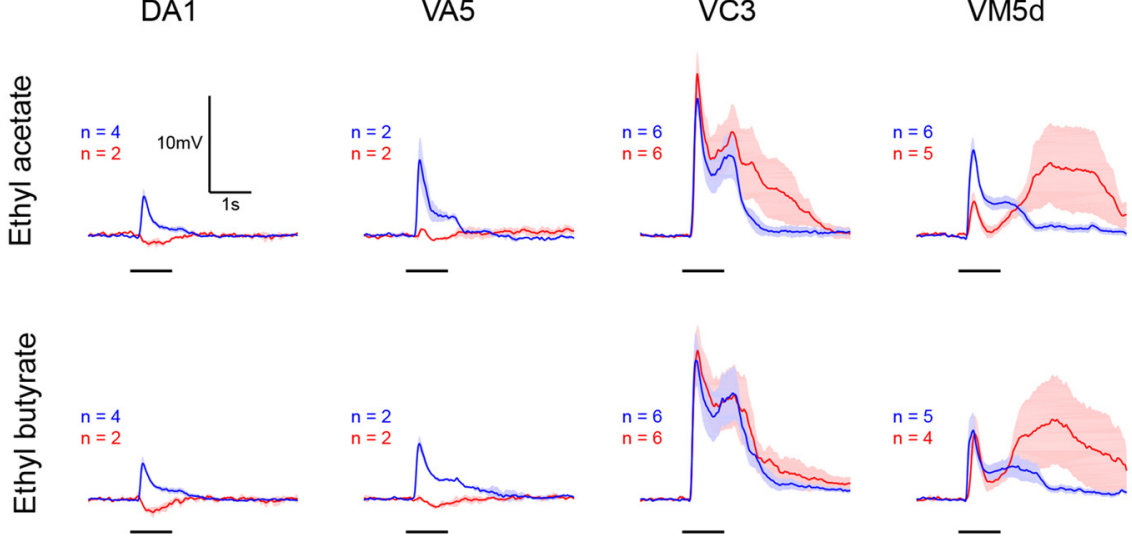

FIGURE 5 | shakB ${ }^{2}$ mutation does not abolish lateral excitatory inputs in all glomeruli. (A) A schematic of the antenna-less preparation. Both antennae were removed immediately before experiments, maxillary palps were stimulated with odors, and patch recordings were performed from antennal ePNs lacking direct afferent inputs. (B) In antenna-less wild-type flies, odors evoked lateral excitation in ePNs in these four glomeruli (blue, mean \pm SEM). While in DA1 and VA5 glomeruli of antenna-less shakB ${ }^{2}$ mutant flies, these odor-evoked lateral excitatory inputs were abolished and inhibition dominated, ePNs in VC3 and VM5d of shak $B^{2}$ mutant flies showed large lateral excitatory inputs (red, mean \pm SEM).

His-Cl channels in MZ699-Gal4 neurons. Prior work showed that application of histamine by itself does not affect the odor responses, resting potentials, or input resistance of ePNs in Drosophila (Liu and Wilson, 2013b). We found that suppressing vPNs by providing histamine in the bath did not reduce the amplitudes of odor-evoked EPSPs in ePNs (Figures 6A-C, $6 \mathrm{ePNs}$ in 4 glomeruli, 7 odor-ePN pairs, $p=0.23$ for the peak amplitude, $p=0.14$ for the area under the curve, paired $t$-test). We also tested whether odor-elicited responses of ePNs in animals with intact antennae and maxillary palps were reduced by suppressing MZ699-vPNs. Though decreases both in peak response spike rate and total response spike count were seen in a few odor-ePN pairs, the overall trend across many glomeruli was that suppression of MZ699-vPNs did not change the odor response of ePNs (Figures 6D-G, 13 ePNs, 32 odor-ePN pairs, $p=0.53$ for the peak response rate, $p=0.13$ for the total response spike count). An increase in odor responses in some glomeruli is consistent with our observation that inhibitory local neurons (iLNs) also receive excitatory inputs from MZ699-vPNs (data not shown); decreased activation of iLNs on suppression of MZ699-vPNs may have disinhibited the odor responses of ePNs. These results suggest the MZ699-vPNs are unlikely to underlie wide-spread lateral excitatory interactions in the Drosophila AL.

\section{DISCUSSION}

In the insect olfactory system, odor information detected on the olfactory appendages is processed in the $\mathrm{AL}$ and is then sent to the $\mathrm{MB}$ and $\mathrm{LH}$ through multiple antennal lobe tracts (ALTs, Schachtner et al., 2005). In flies and moths, the mALT targets the MB calyx and the $\mathrm{LH}$, and the mlALT targets only the LH, bypassing the calyx (Homberg et al., 1988; Stocker et al., 1990; Tanaka et al., 2012). In honeybees, on the other hand, the mALT and IALT target the MB calyx and $\mathrm{LH}$, and the mlALT directly targets the $\mathrm{LH}$ (Kirschner et al., 2006). In all these species, despite the slight differences in the projection patterns of ALTs, a substantial proportion of mlALT neurons are GABAergic (Hoskins et al., 1986; Schäfer and Bicker, 1986; Okada et al., 2009). In Drosophila, in situ hybridization analysis and genetic labeling strategies have revealed that about $80 \%$ of MZ699 expressing vPNs are GABAergic, and appear to have relatively few presynaptic sites in the AL (Okada et al., 2009; Liang et al., 2013). These observations have helped draw attention to the roles inhibition from vPNs may play upon their postsynaptic partners in the LH (Liang et al., 2013; Parnas et al., 2013; Wang et al., 2014). However, very little is known about the properties and functions of synapses vPNs make with their dendritic branches in the AL although it has been reported that vPNs form weak electrical synapses with ePNs through gap junctions (Wang et al., 2014).

We used optogenetic and pharmacological manipulations to investigate synapses between vPNs and ePNs in the AL. We found that in the AL, vPNs labeled by the Gal4 driver MZ699-Gal4 form monosynaptic, excitatory chemical synapses onto ePNs. When driven optogenetically, vPNs depolarize ePNs in several glomeruli, with the amplitudes of the EPSPs relatively large and varying with the glomerulus (Figure 1D). MZ699Gal4 is expressed not only in vPNs but also in neurons in other locations in the brain, including the vlpr neurons and two fibers innervating the AL, both of which could potentially make direct contacts with ePNs (Figures 1A,B, 2E1,E2). However, by selectively illuminating different brain 


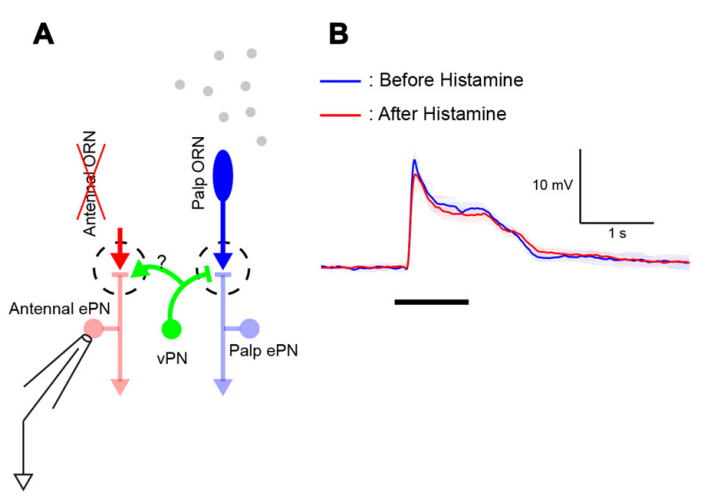

C

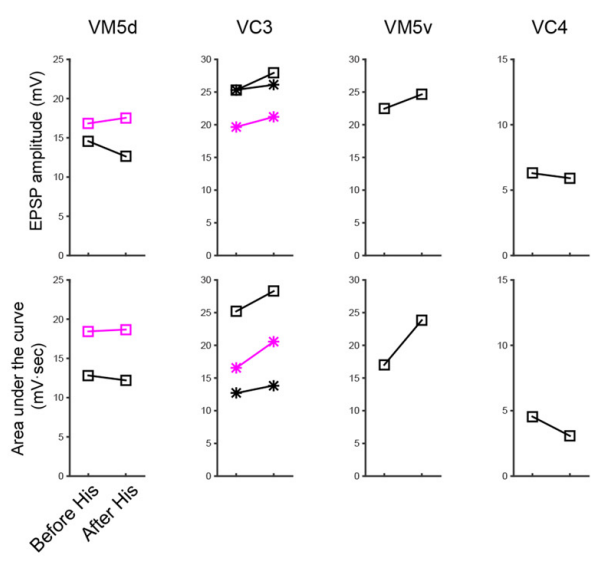

D

E

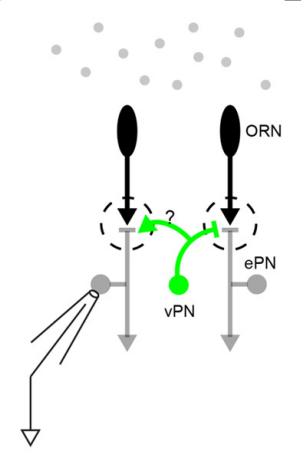

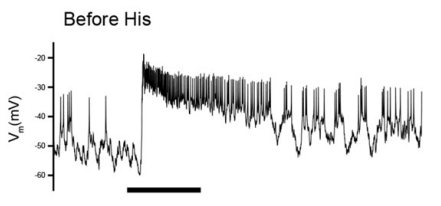

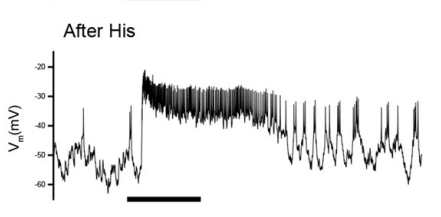

$\mathbf{F}$
— : Before Histamine

— : After Histamine $\square$ : Ethyl butyrate

* : Ethyl acetate

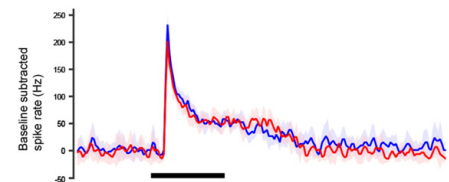

G

VA7I

DL2v

VC3
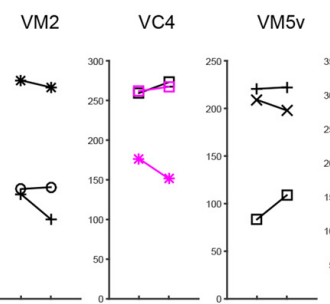

VM3

VM5d
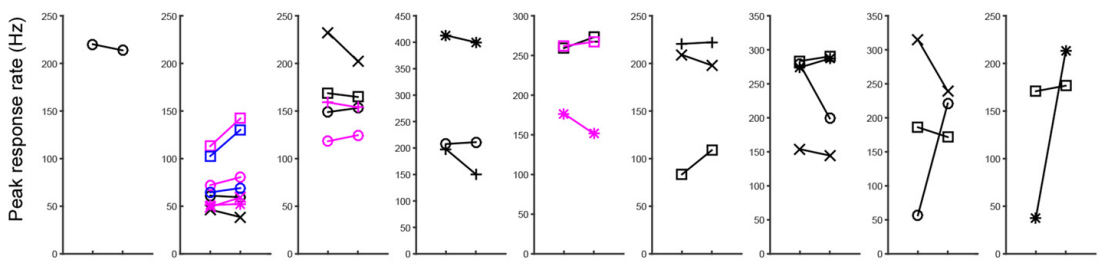

$\mathrm{O}:$ Benzaldehyde

$+:$-Octanone

$X$ : Pentyl acetate

* : Ethyl acetate

$\square$ : Ethyl butyrate
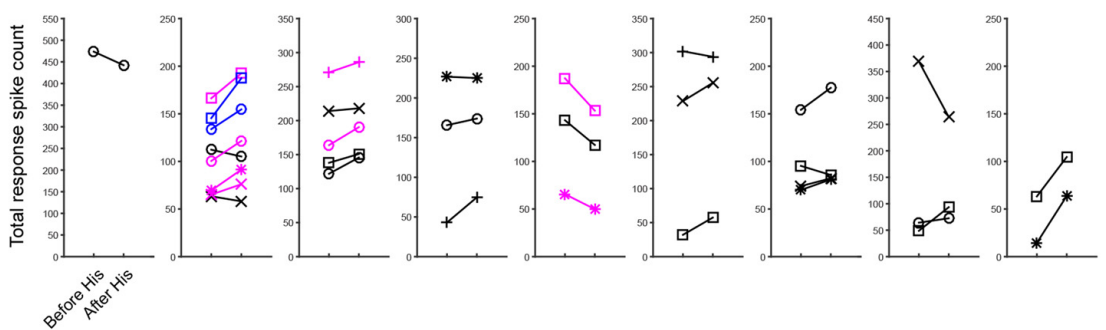

FIGURE 6 | Widespread lateral excitation is not affected by suppressing vPNs. (A) A schematic of the antenna-less preparation. (B) Across-trial average of odor-elicited EPSPs from an example VM5d ePN (black plots in VM5d panel (C)) before and after suppressing vPNs expressing shunting His-Cl channels by adding histamine to the bath (mean \pm SD) suggests that the vPN-ePN synapse does not contribute to lateral excitation. Odor stimulus in this example was a $1 \mathrm{~s}$ puff of $0.3 \%$ ethyl butyrate. (C) Results from similar experiments (six ePNs, seven odor-ePN pairs, $p=0.23$ for the peak amplitude, $p=0.14$ for the area under the curve, two-way ANOVA). Different ePNs in each glomerulus are indicated with different colors (black or magenta). (D) A schematic of the intact preparation. Both antennae and maxillary palps were stimulated with odors while ePNs were monitored with patch recordings. (E) Example voltage traces show odor-elicited responses in a VC3 ePN (black plots in VC3 panel (G)) before and after vPNs were suppressed by His-Cl channels. Odor stimulus was $1 \mathrm{~s}$ of $0.3 \%$ pentyl acetate. (F) Across-trial average of PSTHs from the same VC3 ePN shown in (E; mean \pm SD). (G) Odor-elicited spiking responses in the palp ePNs (VA7l) and antennal ePNs (all others) were not reduced when vPNs were suppressed by His-Cl channels ( $13 \mathrm{ePNs}$, 32 odor-ePN pairs, $p=0.53$ for the peak response rate, $p=0.13$ for the total response spike count, two-way ANOVA, see "Materials and Methods" Section for response calculations). Different ePNs in each glomerulus are indicated with different colors (black, magenta or blue). 
areas and restricting CsChrimson expression through a genetic intersectional approach, we determined that the MZ699-vPNs are the dominant source of excitation to the ePNs (Figure 2). We further determined that connections from MZ699-vPNs to ePNs were monosynaptic by measuring the time between the onset of photoactivating light flashes and the onset of purely chemical component of EPSPs in ePNs $(<1.5 \mathrm{~ms})$, and by comparing the light-evoked EPSP amplitude before and after blocking action potentials with bath-applied TTX (Figure 3).

Although GABA most often mediates inhibition, it is known to play excitatory roles in some contexts, such as in immature mammalian brains, in worms, and in the oocyte heterologous expression system with Drosophila GRD/LCCH3 (Beg and Jorgensen, 2003; Gisselmann et al., 2004; Raimondo et al., 2017). However, multiple lines of evidence suggest GABAergic transmission cannot explain synaptic excitation in the MZ699-vPNs to ePNs synapses (Figure 4): (1) the amplitude of EPSPs in ePNs on activation of MZ699-vPNs was not reduced by application of PTX, which effectively blocks both $\mathrm{GABA}_{\mathrm{A}}$ receptors and GRD-LCCH3 cation channels in Drosophila; (2) RNAi knock down of the GABA-generating enzyme Gad1 in MZ699-Gal4 positive cells did not reduce EPSP magnitude in ePNs; and (3) exogenous application of GABA pulses to the AL in the presence of $\mathrm{GABA}_{\mathrm{A}}$ and $\mathrm{GABA}_{\mathrm{B}}$ antagonist never induced depolarization in ePNs.

About 20\% of MZ699-vPNs are unlikely to be GABAergic because they were not labeled by a DNA in situ hybridization probe against Gad1 mRNA (Okada et al., 2009). If these vPNs express an excitatory neurotransmitter whose synaptic release activates corresponding receptors in the ePNs, the excitatory vPN-ePN synaptic connections we observed could potentially be mediated by these vPNs. Glutamate and acetylcholine are the most common fast neurotransmitters in the insect peripheral and central nervous system, respectively (Osborne, 1996). In Drosophila, however, iontophoresis of glutamate into the AL has been shown previously to inhibit the ePNs through glutamategated $\mathrm{Cl}^{-}$channels (Liu and Wilson, 2013a). We further found that bath application of mecamylamine (a nicotinic acetylcholine receptor antagonist) did not reduce depolarization of ePNs upon optogenetic activation of MZ699-vPNs (data not shown, although other cholinergic receptors insensitive to mecamylamine could mediate excitatory cholinergic transmission from vPNs to ePNs). It is also possible that excitatory transmitters other than glutamate or acetylcholine that are not well characterized in Drosophila may contribute to the depolarization of ePNs. Identification of expression lines that specifically label GABAergic or non-GABAergic subset of vPNs will allow further insights into synaptic mechanisms underlying the excitatory connection from vPNs to ePNs.

A possibility we cannot rule out is that GABAergic MZ699-vPNs could co-express an excitatory neurotransmitter and release it at specific compartments within cells; for example, GABA could be released at the axonal terminals in the $\mathrm{LH}$ and an excitatory neurotransmitter released at the dendritic presynaptic terminals in the AL. Indeed, several examples of mammalian neurons that can release multiple fast excitatory or inhibitory neurotransmitters have been reported, such as spatially segregated release of GABA and ACh in the retina (Lee et al., 2010; Vaaga et al., 2014; Granger et al., 2016).

Electrical synapses between krasavietz-Gal4 eLNs and ePNs have been proposed to mediate lateral excitation between glomeruli in the Drosophila AL (Yaksi and Wilson, 2010), but several lines of evidence align against this mechanism. Because the number of eLNs is small (two or three), and the coupling reported between each eLN and ePN is weak (1-2 $\mathrm{mV}$ EPSPs), it is not clear how activity mediated by electrical synapses is sufficient to explain the strong odor-evoked lateral excitation observed in some glomeruli ( $\sim 8 \mathrm{mV}$ on average, up to $20 \mathrm{mV}$, Olsen et al., 2007, and Figure 5). In addition, recordings from eLNs in antenna-less preparations revealed odor responses that began with a period of inhibition, while responses of antennal ePNs (now lacking direct afferent input from antennal ORNs) began with a period of excitation (Kazama et al., 2011); if lateral excitation is mediated by the eLN-ePN electrical synapses, the sign of responses in ePNs and eLNs should match: the responses of eLNs also should begin with a period of excitation in the antenna-less preparation. Further, we found that mutants lacking shakB still showed strong odor-elicited lateral excitation in some glomeruli (Figure 5). Because some MZ699-vPNs form multiglomerular arborizations (Lai et al., 2008) and because we observed strong excitatory chemical connections from the MZ699-vPNs to the ePNs, MZ699-vPNs seemed well-positioned to mediate wide-spread lateral excitation. Indeed, a previous study proposed that vPNs could contribute to lateral excitation given that MZ699-vPNs make electrical synapses onto ePNs (Wang et al., 2014). However, we observed reduction of odor-elicited responses in neither antenna-less nor intact preparations when MZ699-vPN activity was suppressed by activating His-Cl channels (Figure 6). Thus, vPN-ePN excitatory connections are unlikely to underlie widespread lateral excitation.

vPN-ePN connections more likely mediate targeted, localized excitation in the AL. One possibility is that the strong synaptic connections revealed by our optogenetic experiments are mediated mostly, if not entirely, by uniglomerular vPNs; synapses from multiglomerular vPNs onto ePNs may be relatively weak; such a connectivity scheme could allow for amplifying the AL's responses to particular odorants. We cannot at present differentiate these populations since the MZ699-vPN population we photoactivated includes both uniglomerular and multiglomerular vPNs. Identification of new Gal4 lines that label morphological subpopulations (uniglomerular or multiglomerular and different glomerular projections) of the vPNs will allow further insights into the roles of this population in olfactory information processing.

\section{AUTHOR CONTRIBUTIONS}

KS and MS: conceptualization; funding acquisition; KS: methodology; investigation; writing-original draft; MS: writing_review and editing; supervision. 


\section{FUNDING}

This work was funded by an intramural grant from the National Institutes of Health-National Institute of Child Health and Human Development (MS). KS was supported by the Japan Society for the Promotion of Science Research Fellowship for Japanese Biomedical and Behavioral Researchers at National Institutes of Health.

\section{REFERENCES}

Beg, A. A., and Jorgensen, E. M. (2003). EXP-1 is an excitatory GABA-gated cation channel. Nat. Neurosci. 6, 1145-1152. doi: 10.1038/nn1136

Berck, M. E., Khandelwal, A., Claus, L., Hernandez-Nunez, L., Si, G., Tabone, C. J., et al. (2016). The wiring diagram of a glomerular olfactory system. Elife 5:e14859. doi: 10.7554/eLife.14859

Couto, A., Alenius, M., and Dickson, B. J. (2005). Molecular, anatomical, and functional organization of the Drosophila olfactory system. Curr. Biol. 15, 1535-1547. doi: 10.1016/j.cub.2005.07.034

Franks, K. M., Russo, M. J., Sosulski, D. L., Mulligan, A. A., Siegelbaum, S. A., and Axel, R. (2011). Recurrent circuitry dynamically shapes the activation of piriform cortex. Neuron 72, 49-56. doi: 10.1016/j.neuron.2011.08.020

Gisselmann, G., Plonka, J., Pusch, H., and Hatt, H. (2004). Drosophila melanogaster GRD and LCCH3 subunits form heteromultimeric GABA-gated cation channels. Br. J. Pharmacol. 142, 409-413. doi: 10.1038/sj.bjp.0705818

Granger, A. J., Mulder, N., Saunders, A., and Sabatini, B. L. (2016). Cotransmission of acetylcholine and GABA. Neuropharmacology 100, 40-46. doi: 10.1016/j. neuropharm.2015.07.031

Homberg, U., Montague, R. A., and Hildebrand, J. G. (1988). Anatomy of antennocerebral pathways in the brain of the sphinx moth Manduca sexta. Cell Tissue Res. 254, 255-281. doi: 10.1007/bf00225800

Hosie, A. M., Aronstein, K., Sattelle, D. B., and ffrench-Constant, R. H. (1997). Molecular biology of insect neuronal GABA receptors. Trends Neurosci. 20, 578-583. doi: 10.1016/s0166-2236(97)01127-2

Hoskins, S. G., Homberg, U., Kingan, T. G., Christensen, T. A., and Hildebrand, J. G. (1986). Immunocytochemistry of GABA in the antennal lobes of the sphinx moth Manduca sexta. Cell Tissue Res. 244, 243-252. doi: 10.1007/bf00219199

Kazama, H., Yaksi, E., and Wilson, R. I. (2011). Cell death triggers olfactory circuit plasticity via glial signaling in Drosophila. J. Neurosci. 31, 7619-7630. doi: 10.1523/JNEUROSCI.5984-10.2011

Kirschner, S., Kleineidam, C. J., Zube, C., Rybak, J., Grünewald, B., and Rössler, W. (2006). Dual olfactory pathway in the honeybee, Apis mellifera. J. Comp. Neurol. 499, 933-952. doi: 10.1002/cne.21158

Kohara, K., Pignatelli, M., Rivest, A. J., Jung, H. Y., Kitamura, T., Suh, J., et al. (2013). Cell type-specific genetic and optogenetic tools reveal hippocampal CA2 circuits. Nat. Neurosci. 17, 269-279. doi: 10.1038/nn.3614

Lai, S. L., Awasaki, T., Ito, K., and Lee, T. (2008). Clonal analysis of Drosophila antennal lobe neurons: diverse neuronal architectures in the lateral neuroblast lineage. Development 135, 2883-2893. doi: 10.1242/dev.024380

Larsson, M. C., Domingos, A. I., Jones, W. D., Chiappe, E., Amrein, H., and Vosshall, L. B. (2004). Or83b encodes a broadly expressed odorant receptor essential for Drosophila olfaction. Neuron 43, 703-714. doi: 10.1016/j.neuron. 2004.08.019

Lee, S., Kim, K., and Zhou, Z. J. (2010). Role of ACh-GABA co-transmission in detecting image motion and motion direction. Neuron 68, 1159-1172. doi: 10.1016/j.neuron.2010.11.031

Liang, L., Li, Y., Potter, C. J., Yizhar, O., Deisseroth, K., Tsien, R. W., et al. (2013). GABAergic projection neurons route selective olfactory inputs to specific higher-order neurons. Neuron 79, 917-931. doi: 10.1016/j.neuron.2013.06.014

Liang, L., and Luo, L. (2010). The olfactory circuit of the fruit fly Drosophila melanogaster. Sci. China Life Sci. 53, 472-484. doi: 10.1007/s11427-010-0099-Z

Liu, W. W., and Wilson, R. I. (2013a). Glutamate is an inhibitory neurotransmitter in the Drosophila olfactory system. Proc. Natl. Acad. Sci. U S A 110, 10294-10299. doi: 10.1073/pnas.1220560110

\section{ACKNOWLEDGMENTS}

The authors thank Dr. Konrad Basler, Dr. Chris Elliott, Dr. Chi-hon Lee, Dr. Tzumin Lee, Dr. Benjamin White, Bloomington Stock Center and Vienna Drosophila Resource Center for fly stocks, and the Developmental Studies Hybridoma Bank for the nc82 antibody. We thank members of the Stopfer lab for helpful discussions and comments.

Liu, W. W., and Wilson, R. I. (2013b). Transient and specific inactivation of Drosophila neurons in vivo using a native ligand-gated ion channel. Curr. Biol. 23, 1202-1206. doi: 10.1016/j.cub.2013.05.016

Neuhaus, E. M., Gisselmann, G., Zhang, W., Dooley, R., Störtkuhl, K., and Hatt, H. (2005). Odorant receptor heterodimerization in the olfactory system of Drosophila melanogaster. Nat. Neurosci. 8, 15-17. doi: 10.1038/ nn1371

Okada, R., Awasaki, T., and Ito, K. (2009). Gamma-aminobutyric acid (GABA)mediated neural connections in the Drosophila antennal lobe. J. Comp. Neurol. 514, 74-91. doi: 10.1002/cne.21971

Olsen, S. R., Bhandawat, V., and Wilson, R. I. (2007). Excitatory interactions between olfactory processing channels in the Drosophila antennal lobe. Neuron 54, 89-103. doi: 10.1016/j.neuron.2007.05.006

Olsen, S. R., and Wilson, R. I. (2008). Lateral presynaptic inhibition mediates gain control in an olfactory circuit. Nature 452, 956-960. doi: 10.1038/ nature 06864

Osborne, R. H. (1996). Insect neurotransmission: neurotransmitters and their receptors. Pharmacol. Ther. 69, 117-142. doi: 10.1016/0163-7258(95) 02054-3

Parnas, M., Lin, A. C., Huetteroth, W., and Miesenböck, G. (2013). Odor discrimination in Drosophila: from neural population codes to behavior. Neuron 79, 932-944. doi: 10.1016/j.neuron.2013.08.006

Raimondo, J. V., Richards, B. A., and Woodin, M. A. (2017). Neuronal chloride and excitability - the big impact of small changes. Curr. Opin. Neurobiol. 43 , 35-42. doi: 10.1016/j.conb.2016.11.012

Root, D. H., Mejias-Aponte, C. A., Zhang, S., Wang, H. L., Hoffman, A. F., Lupica, C. R., et al. (2014). Single rodent mesohabenular axons release glutamate and GABA. Nat. Neurosci. 17, 1543-1551. doi: 10.1038/ nn.3823

Rytz, R., Croset, V., and Benton, R. (2013). Ionotropic receptors (IRs): chemosensory ionotropic glutamate receptors in Drosophila and beyond. Insect Biochem. Mol. Biol. 43, 888-897. doi: 10.1016/j.ibmb.2013. 02.007

Schachtner, J., Schmidt, M., and Homberg, U. (2005). Organization and evolutionary trends of primary olfactory brain centers in Tetraconata (Crustacea+Hexapoda). Arthropod Struct. Dev. 34, 257-299. doi: 10.1016/j.asd. 2005.04.003

Schäfer, S., and Bicker, G. (1986). Distribution of GABA-like immunoreactivity in the brain of the honeybee. J. Comp. Neurol. 246, 287-300. doi: 10.1002/cne. 902460302

Sodickson, D. L., and Bean, B. P. (1996). GABA $B$ receptor-activated inwardly rectifying potassium current in dissociated hippocampal CA3 neurons. J. Neurosci. 16, 6374-6385.

Stocker, R. F., Lienhard, M. C., Borst, A., and Fischbach, K. F. (1990). Neuronal architecture of the antennal lobe in Drosophila melanogaster. Cell Tissue Res. 262, 9-34. doi: 10.1007/bf00327741

Sugimura, Y. K., Takahashi, Y., Watabe, A. M., and Kato, F. (2016). Synaptic and network consequences of monosynaptic nociceptive inputs of parabrachial nucleus origin in the central amygdala. J. Neurophysiol. 115, 2721-2739. doi: 10.1152/jn.00946.2015

Tanaka, N. K., Suzuki, E., Dye, L., Ejima, A., and Stopfer, M. (2012). Dye fills reveal additional olfactory tracts in the protocerebrum of wild-type Drosophila. J. Comp. Neurol. 520, 4131-4140. doi: 10.1002/cne.23149

Vaaga, C. E., Borisovska, M., and Westbrook, G. L. (2014). Dual-transmitter neurons: functional implications of co-release and co-transmission. Curr. Opin. Neurobiol. 29, 25-32. doi: 10.1016/j.conb.2014.04.010 
Vosshall, L. B., Wong, A. M., and Axel, R. (2000). An olfactory sensory map in the fly brain. Cell 102, 147-159. doi: 10.1016/s0092-8674(00)00021-0

Wang, K., Gong, J., Wang, Q., Li, H., Cheng, Q., Liu, Y., et al. (2014). Parallel pathways convey olfactory information with opposite polarities in Drosophila. Proc. Natl. Acad. Sci. U S A 111, 3164-3169. doi: 10.1073/pnas. 1317911111

Wilson, R. I. (2013). Early olfactory processing in Drosophila: mechanisms and principles. Annu. Rev. Neurosci. 36, 217-241. doi: 10.1146/annurev-neuro062111-150533

Wilson, R. I., and Laurent, G. (2005). Role of GABAergic inhibition in shaping odor-evoked spatiotemporal patterns in the Drosophila antennal lobe. J. Neurosci. 25, 9069-9079. doi: 10.1523/JNEUROSCI.2070-05.2005

Wong, A. M., Wang, J. W., and Axel, R. (2002). Spatial representation of the glomerular map in the Drosophila protocerebrum. Cell 109, 229-241. doi: 10.1016/s0092-8674(02)00707-9

Yaksi, E., and Wilson, R. I. (2010). Electrical coupling between olfactory glomeruli. Neuron 67, 1034-1047. doi: 10.1016/j.neuron.2010.08.041
Yu, H. H., Kao, C. F., He, Y., Ding, P., Kao, J. C., and Lee, T. (2010). A complete developmental sequence of a Drosophila neuronal lineage as revealed by twin-spot MARCM. PLoS Biol. 8:e1000461. doi: 10.1371/journal.pbio.1000461

Zhang, Z., Curtin, K. D., Sun, Y. A., and Wyman, R. J. (1999). Nested transcripts of Gap junction gene have distinct expression patterns. J. Neurobiol. 40, 288-301. doi: 10.1002/(SICI)1097-4695(19990905)40:3<288::aid-neu2>3.0.co;2-o

Conflict of Interest Statement: The authors declare that the research was conducted in the absence of any commercial or financial relationships that could be construed as a potential conflict of interest.

Copyright $\odot 2017$ Shimizu and Stopfer. This is an open-access article distributed under the terms of the Creative Commons Attribution License (CC BY). The use, distribution or reproduction in other forums is permitted, provided the original author(s) or licensor are credited and that the original publication in this journal is cited, in accordance with accepted academic practice. No use, distribution or reproduction is permitted which does not comply with these terms. 